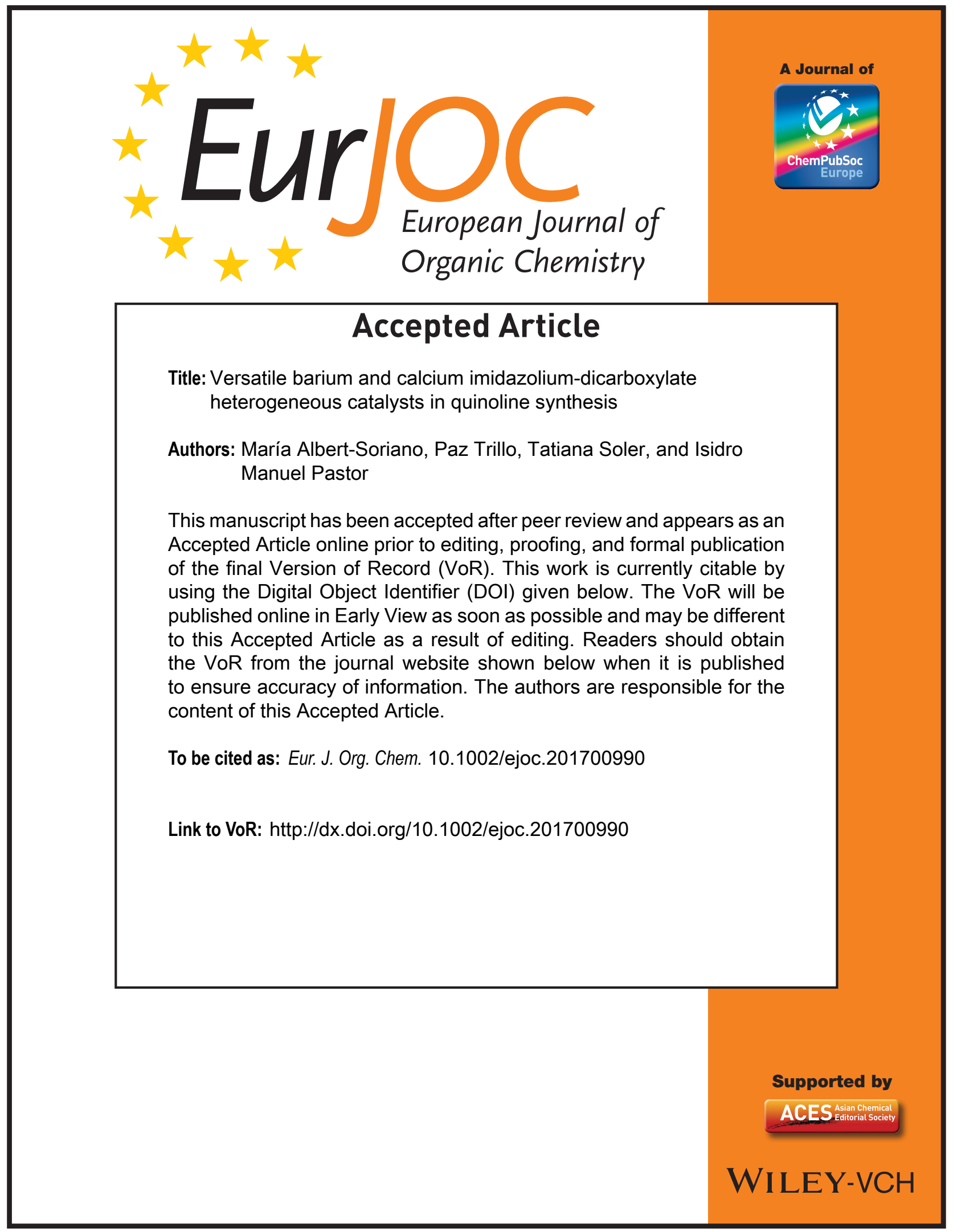




\title{
Versatile barium and calcium imidazolium-dicarboxylate heterogeneous catalysts in quinoline synthesis
}

\author{
María Albert-Soriano,,$^{[a][t]}$ Paz Trillo, ${ }^{[a][b][t]}$ Tatiana Soler, ${ }^{[c]}$ and Isidro M. Pastor ${ }^{\star[a]}$ \\ Dedication ((optional))
}

\begin{abstract}
This article details the development of heterogeneous catalysts based on calcium and barium in combination with the organic linker 1,3-bis(carboxymethyl)imidazolium (bcmim). The linker and the materials from alkaline earth metals are easily prepared under very smooth conditions. The use of linkers with different counterions $(\mathrm{Cl}$ or $\mathrm{Br})$ provided different materials. Calcium and barium based catalysts were successfully employed in the preparation of quinoline derivatives from ketones and 2aminoarylaldehydes or 2-aminoarylketones. Moreover, barium based catalysts provided better results than calcium, although the latter are an excellent complement for certain substrates. Thus, a notable feature of such catalysts is the possibility of accessing a variety of complementary heterogeneous catalytic systems, rendering the catalysis adaptive to the reactant.
\end{abstract}

\section{Introduction}

Quinoline moiety is present in several bioactive compounds, both natural occurring and synthetic. The spectrum of pharmacological applications covers different aspects, such as antifungal, antiviral, antibacterial, antimalarial, antiplatelet aggregation and anti-inflammatory among others. ${ }^{[1]}$ More recently, quinoline derivatives have shown anticancer activity by means of different mechanisms of action. ${ }^{[2]}$ In this sense, the study of new approaches and/or protocols for the preparation of this type of heterocyclic derivatives is of relevant interest. One of the most employed routes in the preparation of quinoline derivatives is the Friedländer reaction. ${ }^{[3]}$ Thus, the condensation between a carbonyl compound bearing hydrogens in the $a$ -

[a] M. Albert-Soriano, Dr. P. Trillo, Dr. I. M. Pastor Organic Chemistry Dpt. and Instituto Síntesis Orgánica (ISO) University of Alicante

Apdo. 99, 03080, Alicante (Spain)

E-mail:paz.trillo@ua.es,maria.albert@ua.es,ipastor@ua.es http://www.researcherid.com/rid//K-7784-2014

ORCID: orcid.org/0000-0003-2351-6790 (M. Albert-Soriano); orcid.org/0000-0002-8271-0641 (I. M. Pastor)

[b] Dr. P. Trillo (current address)

Chemistry Dpt., University of Umeå

90187, Umeå (Sweden)

E-mail: paz.trillo@umu.se

ORCID: orcid.org/0000-0001-9540-630X (P. Trillo)

[c] Dr. T. Soler

$X$-rays of monocristal and vibracional

and optical spectroscopies Unit, Research Technical Services

University of Alicante

Apdo. 99, 03080, Alicante (Spain)

E-mail: tatiana.soler@ua.es

[†] These authors contributed equally to this work

Supporting information for this article is given via a link at the end of the document. position and a 2-aminoaryl ketone or aldehyde allows the formation of the nitrogen-containing heterocycle in one synthetic step. The reaction can be either acid or base catalyzed. ${ }^{[4]}$ Among the catalysts considered in this transformation, no alkaline earth metal based ones have been tested. ${ }^{[5]}$ Regarding heterogeneous catalysts, alumina or acidic supported materials have been reported. ${ }^{[3]}$ The use of metal-organic frameworks (MOF) as heterogeneous catalysts is nowadays of particular interest due to their specific characteristics. ${ }^{[6]}$ Thus, the group of Cejka evidenced the idea that a metal-organic framework (MOF) can act as heterogeneous catalyst in the Friedländer reaction. Copper-MOF Basolite C-300 (HKUST-1) catalysed the coupling between pentane-2,4-dione and 2-aminobenzophenone or 2aminoacetophenone..$^{[7]}$ Other copper based MOF, such as MOF505 and MMCF-2, have been also proved to be active in the condensation between 2-aminobenzophenone and pentane-2,4dione or ethyl 3-oxobutanoate. ${ }^{[8]}$ The concept of MOFs acting as heterogeneous catalysts in this transformation has been just shown, albeit the catalyst has a lack of versatility in terms of reactants. Furthermore, the reuse of a heterogeneous catalyst is one of its important features, what can be a challenge in this type of transformation. Quinolines are nitrogen-containing compounds, which can be adsorptive removed from fuel and air by metal-organic frameworks..$^{[9]}$ Consequently, the product of the reaction may cause deactivation of the catalyst, reducing catalyst recyclability.

Catalysis employing metal complexes is mainly based on transition metals, and the development of this area has been essential for synthetic organic chemistry. The use of alkaline earth metals, such as calcium and barium, ${ }^{[10]}$ in catalytic systems has been surprisingly less studied, despite their economic and ecological benefits. There are few examples of the evidence of calcium or barium based hybrid materials ${ }^{[11]}$ as heterogeneous catalysts, being just a simple test of the catalytic activity reported in each case. The group of Koner has described two barium-organic frameworks using pyridine-2,5dicarboxylate $^{[12]}$ and 3,5-pyrazoledicarboxylate ${ }^{[13]}$ as linkers, which have been employed as catalysts for the aldol condensation of various aromatic aldehydes with acetone and cyclohexanone with high yields and selectivities. Hybrid materials based on barium and 1,3,5-benzenetricarboxylate (BTC) have proved to be basic catalysts in Knoevenagel condensation and Michael addition reactions. The catalytic activity could be increased when they were pretreated at $320 \stackrel{\circ}{\circ} \mathrm{C}$ to generate new active sites. ${ }^{[14]}$ Barium linked with $5,5^{\prime}-(2,3,6,7-$ tetramethoxyanthracene-9,10-diyl)diisophthalate has been tested in the cyanosilylation of benzaldehydes and phenones and in the cycloaddition of carbon dioxide and epoxides. ${ }^{[15]}$ The group of Monge and Gutiérrez-Puebla has employed the linker anthraquinone-2,6-disulfonate with calcium and barium to generate heterogeneous catalysts for styrene hydrogenation and 
diphenylsilylation of benzaldehyde. ${ }^{[16]}$ Additionally, calcium has been linked with 4,4'-(hexafluoroisopropylidene)bis(benzoate) to prepare a catalyst for the styrene hydrogenation, ${ }^{[17]}$ and connected with 5-(1,2,4-triazol-4-yl)isophthalate to obtain a catalyst for the diphenylsilylation of benzaldehyde. ${ }^{[18]}$

In our research group, aware of the importance of finding sustainable processes, we consider significant the search for catalytic systems, both heterogeneous and homogeneous, which allow their profitability. In this sense, functionalized imidazole derivatives can be employed as interesting components for catalytic systems. We have employed functionalized imidazolium derivatives as precursors of $\mathrm{N}$ heterocyclic carbene (NHC) ligands for palladium. ${ }^{[19]}$ They can also be used in combination with metal salts, such as iron(III) chloride, to produce iron based Lewis acid ionic liquids (IBLAILs), which proved to be very versatile catalytic systems. ${ }^{[20]}$ Furthermore, we employed a metal-organic framework based on imidazolium dicarboxylate as efficient and robust catalyst for an oxidative coupling between carboxylic acids and formamides. ${ }^{[21]}$ On the other hand, the use of heterogeneous catalysts based on alkaline earth metals is quite scarce. Thus following our interests, we have studied the catalytic activity of different heterogeneous catalysts based on alkaline earth, such as calcium and barium (bcmim-Ca and bcmim-Ba). In order to compare their activity we have chosen the formation of quinoline derivatives by the condensation reaction between acylanilines and ketones. Additionally, the copper-organic framework (bcmim-Cu) ${ }^{[21]}$ with the same organic linker has been taken into consideration in the study for comparison.

\section{Results and Discussion}

The organic linker 1,3-bis(carboxymethyl)imidazolium $(\text { bcmim })^{[22]}$ is easily prepared from readily accessible starting materials (i.e. formaldehyde, glyoxal and glycine), and it can be transformed into its corresponding halide salts by simple treatment with the corresponding hydrogen halide (Scheme 1). ${ }^{[23]}$ 1,3-Bis (carboxymethyl)imidazolium bromide (bcmim. $\mathbf{H B r}$ ) in combination with basic calcium and barium salts gave the corresponding metal-organic frameworks bcmim-Ca1 and bcmim-Ba1 as colorless solids (Scheme 1 and Supporting Information), as previously reported. ${ }^{[24]}$ In an analogous way, the 1,3-bis (carboxymethyl)imidazolium chloride (bcmim. $\mathbf{H C l}$ ) was treated with calcium carbonate and barium oxide, under very mild conditions, to form the materials bcmim-Ca2 and bcmimBa2, which were isolated also as colorless solids (Scheme 1 and Supporting Information). The obtained bcmim-Ca1, bcmimCa2, bcmim-Ba1 and bcmim-Ba2 were characterized by elemental analysis, inductively coupled plasma optical emission spectrometry (ICP-OES), podwer X-ray diffraction (PXRD), thermogravimetric analysis (TGA), scanning electron microscopy (SEM) with energy dispersive X-ray (EDX) spectroscopy, and Fourier transform infrared (FTIR) spectroscopy (Supporting Information). It is worth noting the ease with which both the ligand (bcmim) and their metallic material derivatives are prepared.

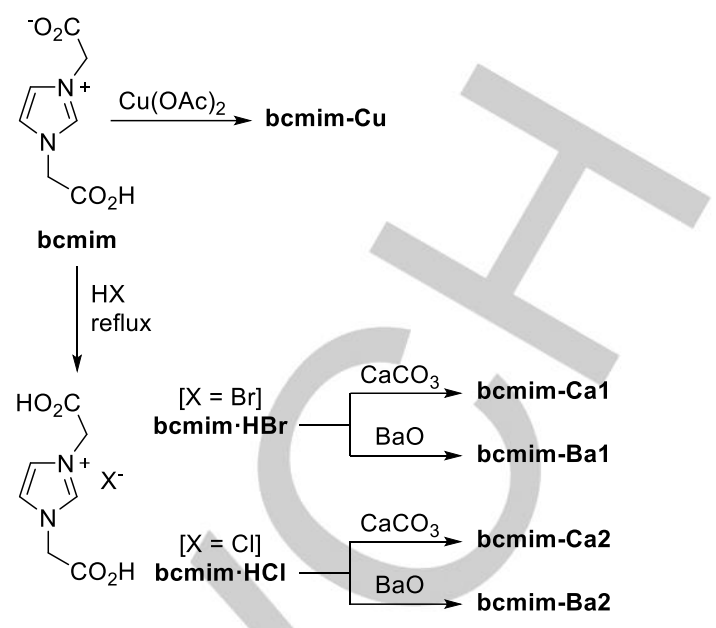

Scheme 1. Preparation of the heterogeneous catalysts.
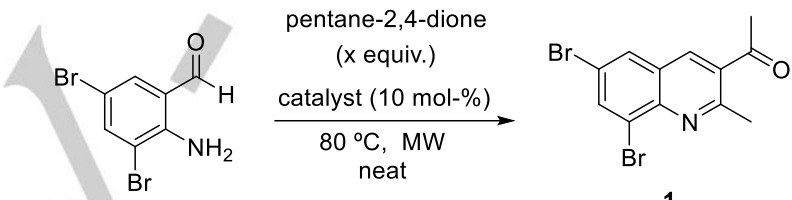

Table 1. Evaluation of heterogeneous catalysts in quinoline synthesis from 3,5-dibromo-2-aminobenzaldehyde. ${ }^{[a]}$

\begin{tabular}{lllll}
\hline Entry & Catalyst $^{[b]}$ & Time $(\min )$. & $x$ & ${\text { Conv. }[\%]^{[c]}}^{(b)}$ \\
\hline 1 & bcmim-Ca1 & 30 & 5 & 34 \\
3 & bcmim-Ca2 & 30 & 5 & 5 \\
\hline 4 & bcmim-Ba1 & 30 & 5 & 31 \\
5 & bcmim-Ba2 & 30 & 5 & 8 \\
6 & bcmim-Cu & 30 & 5 & 43 \\
7 & bcmim-Ca1 & 90 & 5 & 59 \\
8 & bcmim-Ba1 & 90 & 5 & 67 \\
9 & bcmim-Cu & 90 & 5 & 77 \\
10 & bcmim-Ca1 & 90 & 10 & 99 \\
11 & bcmim-Ba1 & 90 & 10 & 65 \\
\hline
\end{tabular}

[a] Reaction conditions: 2-aminoaryl aldehyde (0.2 mmol), pentane-2,4dione ( $x$ equiv.), catalyst (10 mol-\% metal based on ICP analysis), $80 \stackrel{\circ}{\circ} \mathrm{C}$, microwave heating $(80 \mathrm{~W})$. [b] bcmim-Ca1 and bcmim-Ba1 were prepared from $\mathbf{b c m i m} \cdot \mathbf{H B r}$, and bcmim-Ca2 and bcmim-Ba2 were prepared from bcmim. $\mathbf{H C l}$. [c] Conversion was obtained by $\mathrm{GC}$ analysis. In all cases, the selectivity of the reaction towards the formation of the quinoline product is $>99 \%$.

At this point, we decided to test 2-aminobenzaldehydes, which have not been used in the Friedländer reaction catalyzed by 
metal-organic frameworks as catalysts. For catalysts comparison, the coupling between 2-amino-3,5-dibromobenzaldehyde and pentane-2,4-dione was performed at $80 \stackrel{\circ}{\circ}$ using microwave heating during 30 minutes (Table 1). The catalysts bcmim-Ca1, bcmim-Ba1 and bcmim-Cu provided the best results, giving the expected quinoline 1 with 34,31 and $43 \%$ of conversion respectively (Table 1, entries 1, 3 and 5). We observed that the catalysts bcmim-Ca1, bcmim-Ba1 and bcmim-Cu were able to work for longer reaction times, and conversions of 59, 67 and $77 \%$ towards quinoline 1 were obtained after 90 minutes (Table 1 , entries 6-8). The full conversion could be reached with catalyst bcmim-Ca1 when double amount of the diketone was used (Table 1, entry 9). In this case, product 1 was isolated pure with a yield $>99 \%$ by simple filtration, which highlights the potential of this type of catalysts. On the contrary, we observed that the conversion obtained with catalyst bcmim-Ba1 remained roughly in the same level (65\% conversion, Table 1 , entry 10$)$, and with bcmim-Cu was slightly increased giving $81 \%$ after the same reaction time (Table 1, entry 12).

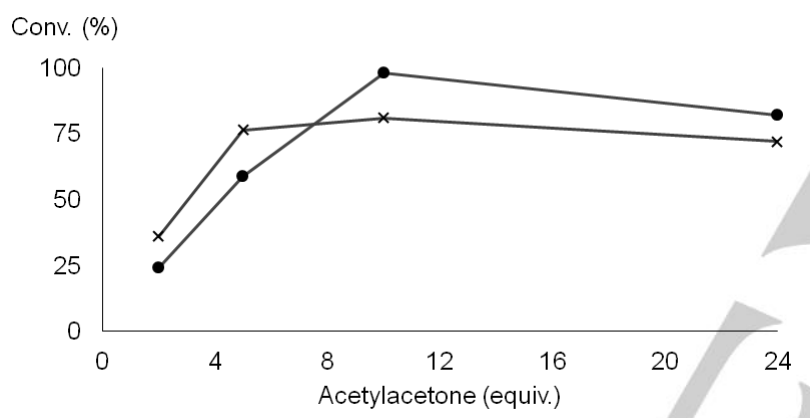

Figure 1. Correlation between the activity of the catalyst and the equivalents of pentane-2,4-dione. $(\bullet)$ for bcmim-Ca1 and $(x)$ for bcmim-Cu. Reaction conditions: 2-aminoaryl aldehyde $(0.2 \mathrm{mmol})$, pentane-2,4-dione ( $x$ equiv.), catalyst (10 mol-\% metal based on ICP analysis), $80 \stackrel{\circ}{\circ}$, microwave heating $(80 \mathrm{~W}), 90 \mathrm{~min}$.

Looking in more detail, an interaction could be observed between the amount of carbonyl compound and the catalysts employed. Indeed, there is a dependence of the conversion on the amount of diketone used (Figure 1), what can be explained by the slowing of the diffusion of the 2-aminobenzaldehyde towards the catalyst. However, the profile obtained for both catalysts is dissimilar, bcmim-Ca1 showing better performance than bcmim-Cu for higher amounts of diketone and vice versa (Figure 1). According to the graph depicted in Figure 1, a slight decrease in yield occurs when the number of equivalents of pentane-2,4-dione is superior to an optimum value. This effect could be observed also for catalyst bcmim-Ba1; the use of an excess of pentane-2,4-dione (20 equiv.) provided quinoline 1 with $61 \%$ yield.

Next, the reaction was performed employing other 2aminobenzaldehydes, ${ }^{[25]}$ using catalyst bcmim-Ca1 under the best reaction conditions, i.e. microwave heating at $80 \stackrel{\circ}{ } \mathrm{C}$ for 90 minutes in the presence of 10 equiv. of pentane-2,4-dione
(Scheme 2). The reaction gave complete conversion when was carried out with 3,5-dibromo-2-aminobenzaldehyde or with 2,4diamino-benzaldehyde, being possible to isolate the corresponding quinolines $\mathbf{1}$ and $\mathbf{4}$ with quantitative yields (Scheme 2). On the other hand, the rest of substrates tested gave the corresponding quinolines with low yield (Scheme 2, compounds 2, $\mathbf{3}$ and $\mathbf{5}$ ). The difference in reactivity cannot be attributed to electronic factors. Thus, best results were obtained with 2-aminobenzaldehydes having electron-withdrawing groups (i.e. bromo) and electron-donating groups (i.e. amino). Moreover, electron-withdrawing groups such as bromo and trifluoromethyl substituents provided very different reactivity. Consequently, the high reactivity of the 2-aminobenzaldehydes containing bromo and amino groups could be due to a possible helpful interaction between those functional groups and the heterogeneous catalyst aiding the approach of the substrate.

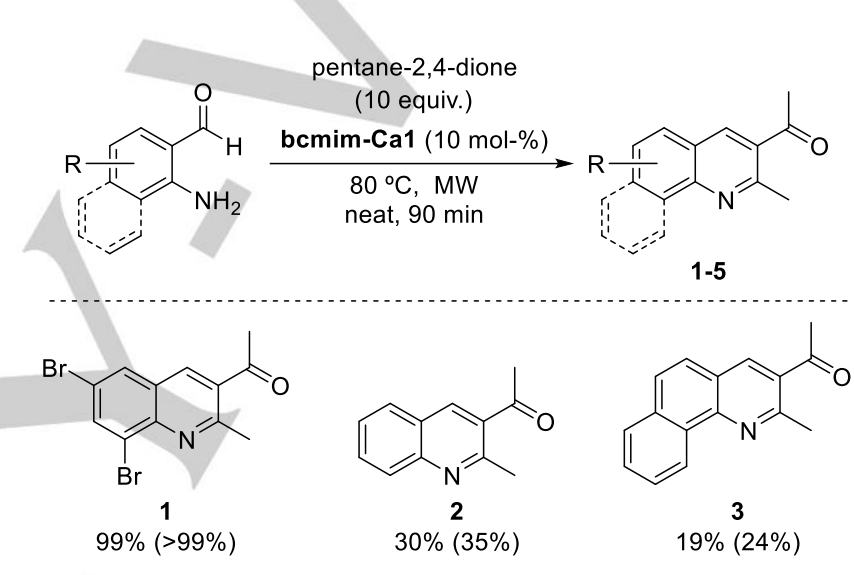<smiles>CC(=O)c1cc2ccc(N)cc2nc1C</smiles><smiles>CC(=O)c1cc2ccc(C(F)(F)F)cc2nc1C</smiles>

Scheme 2. Scope evaluation of 2-aminoaryl aldehydes using bcmim-Ca1 Reagents and conditions: 2-aminoaryl aldehyde $(0.2 \mathrm{mmol})$ and pentane-2,4 dione $(2 \mathrm{mmol})$, bcmim-Ca1 (10 mol-\% metal based on ICP analysis), $80 \stackrel{\circ}{\circ} \mathrm{C}$, microwave heating $(80 \mathrm{~W}), 90 \mathrm{~min}$. Yields are of isolated products. The conversions by GC analysis are reported in parentheses.

From the first use of metal-organic frameworks as heterogeneous catalysts, ${ }^{[26]}$ the importance of the shape and size of the substrate has been documented. Taking the advantage of the readily availability of the studied heterogeneous catalysts, we could take the possibility of improving the results for one of the non-suitable substrates just by changing the catalyst. Thus, bcmim-Cu catalysed the formation of quinoline 2 with $78 \%$ yield (Table 2). Even more interesting was the conversion (97\%) obtained when the reaction was carried out in the presence of bcmim-Ba1 (Table 2). Moreover, similar results were obtained for this catalyst with 2 or 5 equivalents of $\beta$-diketone, thus we performed the reaction with 2 equivalents. Positively, the catalyst bcmim-Ba1 allowed 
improving the previous results obtained with the less active aminoaryl aldehydes (Table 2).

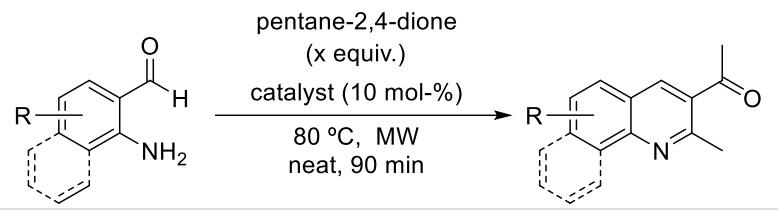

Table 2. Evaluation of catalysts bcmim-Ba1 and bcmim-Cu in quinoline synthesis from 2-aminobenzaldehydes ${ }^{[a]}$

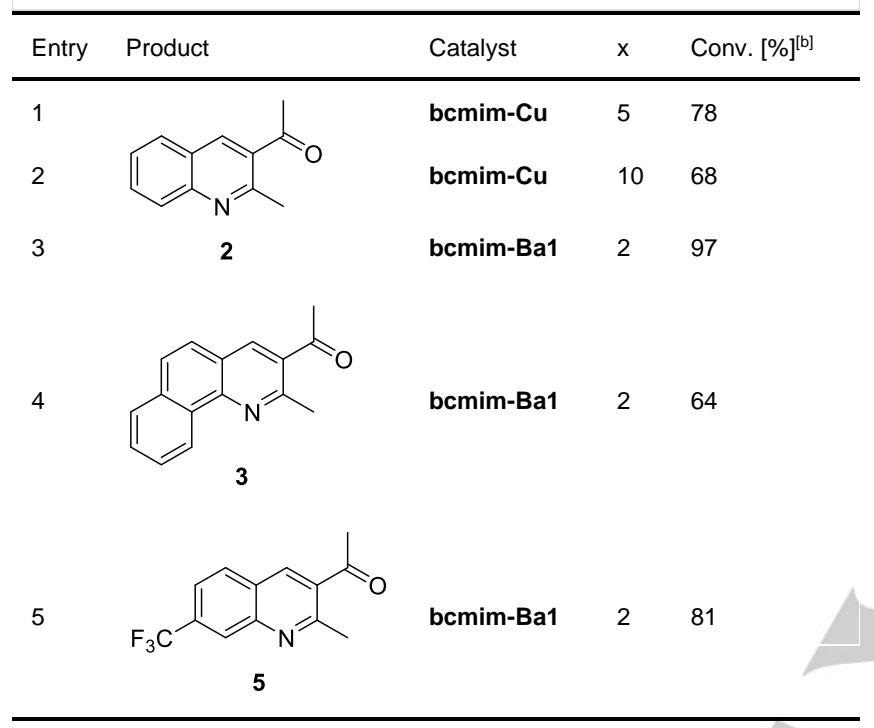

[a] Reaction conditions: 2-aminoaryl aldehyde $(0.3 \mathrm{mmol})$, pentane-2,4dione (x equiv.), catalyst (10 mol-\% metal based on ICP analysis), $80 \stackrel{\circ}{\circ}$, microwave heating $(80 \mathrm{~W}), 90 \mathrm{~min}$. [b] Conversion was obtained by GC analysis. In all cases, the selectivity of the reaction towards the formation of the quinoline product is $>99 \%$.

In order to continue our study, we considered the use of aminoaryl ketones as starting materials, which have been more generally used with other catalytic systems. The condensation of 2-aminobenzophenone with pentane-2,4-dione was chosen as the model reaction. Both reactants were heated in the presence of the corresponding heterogeneous catalyst at $80{ }^{\circ} \mathrm{C}$ using microwave heating (irradiation of $80 \mathrm{~W}$, Table 3). All the catalysts provided conversion over $80 \%$, although catalysts bcmim-Ca1 and bcmim-Ba1 performed slightly better than their analogous bcmim-Ca2 and bcmim-Ba2 and the corresponding copper catalyst. We also proved that the catalysts gave comparable levels of activity when the reaction is performed using conventional heating ( $80{ }^{\circ} \mathrm{C}$, oil bath, Table 3 ), but the reaction times are longer.

The reaction mixture was separated from the solid catalyst by simple decantation using ethyl acetate. The catalyst could be reused in a new cycle after rinsing several times with diethyl ether and drying it. Following this simple work up the different catalysts were recycled, although their activity went down a tad in the following cycles (Table 3 ) with the exception of catalyst bcmim-Ba1. In fact, catalyst bcmim-Ba1 could be reused up to four times without appreciable loss of activity (Table 3, entry 3), both under conventional and microwave heating. Partial deactivation of these heterogeneous catalysts could be due to the presence of by-products remaining after the reaction. ${ }^{[21]}$ The standard washing (with EtOAc and $\mathrm{Et}_{2} \mathrm{O}$ ) could be not enough in order to recover the original activity, being necessary another solvents and/or longer rinsing times. Consequently, the bariumorganic framework bcmim-Ba1 resulted the most rugged heterogeneous catalyst among those considered in the present study. Moreover, the organic phases, after separation of the catalysts, were analysed by inductively coupled plasma-optical emission spectrometry (ICP-OES). The filtrates from the reaction with bcmim-Ba1 and bcmim-Ba2 had less than $0.01 \%$ of barium (refer to the catalyst loading), and the filtrates from the reaction with bcmim-Ca1 and bcmim-Ca2 had less than $0.44 \%$ and $0.17 \%$ of calcium respectively (refer to the catalyst loading). Additionally, a reaction catalysed by bcmim-Ba1 was separated from the catalyst when the conversion was $24 \%$, and the filtrate was allowed to react during $6 \mathrm{~h}$, observing that the reaction stopped ( $27 \%$ conversion). This clearly proves that the reaction is heterogeneous catalysed by the MOF.

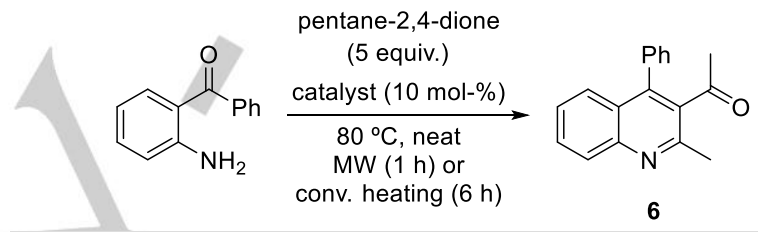

Table 3. Evaluation of heterogeneous catalysts in quinoline synthesis from 2-aminobenzophenone ${ }^{[\mathrm{a}]}$

\begin{tabular}{llll}
\hline & & \multicolumn{2}{c}{ Conversion $[\%]^{[\mathrm{b}]}$} \\
\cline { 3 - 4 } Entry & Catalyst & $\mathrm{MW}, 1 \mathrm{~h}^{[\mathrm{c}]}$ & Conv. heating, $6 \mathrm{~h}^{[\mathrm{c}]}$ \\
\hline 1 & bcmim-Ca1 & $87 / 60$ & $86 / 57 / 50$ \\
2 & bcmim-Ca2 & $81 / 75$ & $73 / 70 / 51$ \\
3 & bcmim-Ba1 & $99 / 99 / 98 / 96$ & $92 / 93 / 92 / 90$ \\
4 & bcmim-Ba2 & $89 / 78$ & $76 / 71 / 67$ \\
5 & bcmim-Cu & $80 / 75$ & $82 / 73 / 59$ \\
\hline
\end{tabular}

[a] Reaction conditions: 2-aminoacetophenone (0.3 mmol), pentane-2,4dione $(1.5 \mathrm{mmol})$, catalyst (10 mol-\% metal based on ICP analysis). [b] Conversion was obtained by GC analysis. In all cases, the selectivity of the reaction towards the formation of the quinoline product is $>99 \%$. [c] Conversion of consecutive catalytic cycles $\left(1^{\text {st }} / 2^{\text {nd }} / 3^{\text {rd }} / 4^{\text {th }}\right)$ with the same catalyst load.

For comparison, we considered the commercially available $\mathrm{Cu}$ BTC MOF (HKUST-1, Basolite C-300), which has been reported previously in the coupling of 2-aminophenones and pentane-2,4dione. The results, with HKUST-1 catalyst, reported in the literature are somewhat different depending on the reaction conditions. ${ }^{[7-8]}$ Therefore, we carried out the reaction with $\mathrm{Cu}$ BTC MOF under the reaction conditions recorded in Table 3 , using conventional heating, obtaining quinoline 6 with $77 \%$ conversion. Thus, the performance of HKUST-1 is in the meanlow of those included in this study, being poorer than bcmimBa1, what remarks the effectiveness of the alkaline earth 
imidazolium-dicarboxylate catalysts. Although, the catalytic activity of the calcium and barium catalysts is taking place in the external surface due to the low surface area and porosity of our catalytic systems.

To extend the versatility of this catalytic system we explored the use of other 2-aminophenones and carbonyl compounds (Scheme 3). In general, the reaction using microwave heating gave comparable to better conversions than the one using conventional heating, and in shorter reaction times. Appropriately, other $\beta$-dicarbonyl compound such as ethyl 3oxobutanoate was coupled with 2-aminobenzophenone giving regioselectively the corresponding quinoline 7 , which could be isolated with $80 \%$ yield. Cyclopentanone and different cyclohexanones reacted smoothly with 2-aminobenzophenone yielding the corresponding quinolines 10-14 (Scheme 3). The use of 2-aminoacetophenone in combination with pentane-2,4dione or cyclohexanone provided the corresponding quinolines 9 and 16 respectively (Scheme 3), even though the conversion was lower than with 2-aminobenzophenone. This observation has been previously reported (with catalyst Cu-BTC MOF), ${ }^{[7}$ although the reaction was expected to work easily. Furthermore, substituted 2-aminobenzophenones were studied as reactants, providing quinolines 8, 15 and $\mathbf{1 7}$ (Scheme 3) with moderate to good conversions and yields. For the latter example, we proved that extending the reaction time the catalyst is still active, being possible to increase the yield. Indeed, the conversion was increased to $90 \%$ after $12 \mathrm{~h}$ of conventional heating, the product being isolated in $81 \%$ yield (Scheme 3 ).

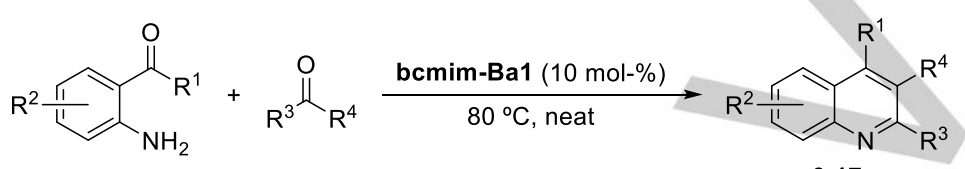

6-17<smiles>CC(=O)c1c(C)nc2ccccc2c1-c1ccccc1</smiles>

6

$90 \%(92 \% / 99 \%)$<smiles>c1ccc(-c2c3c(nc4ccccc24)CCC3)cc1</smiles>

14

$84 \%(81 \% / 99 \%)$<smiles>CCOC(=O)c1c(C)nc2ccccc2c1-c1ccccc1</smiles>

7 $80 \%(90 \% / 99 \%)$<smiles>Clc1ccc2nc3c(c(-c4ccccc4)c2c1)CCCC3</smiles>

15 $86 \%(99 \% / 90 \%)$<smiles>CC(=O)c1c(C)nc2ccc(Cl)cc2c1-c1ccccc1</smiles>

8<smiles>Cc1c2c(nc3ccccc13)CCCC2</smiles>

16 $45 \%(50 \% / 48 \%)$<smiles>CCCCCC1Cc2c1ccc1nc(C)c(C(C)=O)cc21</smiles>

9

$28 \%(29 \% / 61 \%)$<smiles>Clc1ccc2nc3c(c(-c4ccccc4Cl)c2c1)CCCC3</smiles>

17

$50 \%(55 \% / 45 \%)$ $81 \%(90 \%)^{[a]}$

Scheme 3. Scope evaluation of 2-aminoacetophenones and ketones using bcmim-Ba1. Reagents and conditions: 2-aminophenone (0.3 mmol), ketone (1.5 $\mathrm{mmol})$, catalyst (10 mol-\% metal based on ICP analysis), $80 \stackrel{\circ}{\circ}$. Yields are of isolated products, from the reaction performed under conventional heating. The conversions by GC analysis are reported in parentheses (conversion using conventional heating for $6 \mathrm{~h} /$ conversion using microwave heating at $80 \mathrm{~W}$ for $1 \mathrm{~h}$ ). [a] Reaction using conventional heating during $12 \mathrm{~h}$. Yield is of isolated product 17 and in parentheses the conversion.

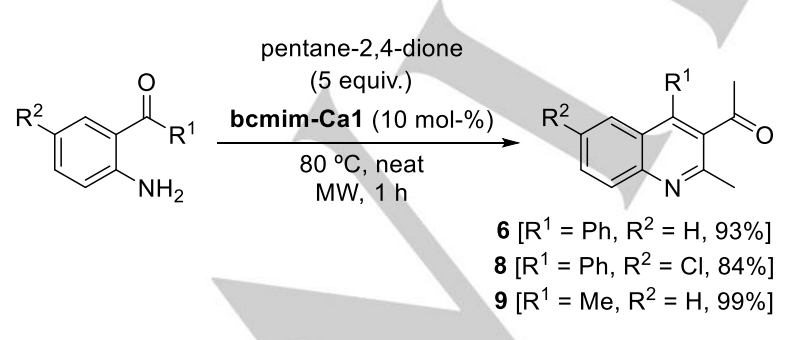

Scheme 4. Reaction of pentane-2,4-dione with 2-aminobenzophenone and 2 aminoacetophenone catalysed by bcmim-Ca1.
As we have commented previously, we have at our disposal a variety of catalysts that allow us to try the improvement of the results. Therefore, 2-amino-5-chlorobenzophenone was reacted with pentane-2,4-dione, using microwave heating, in the presence of bcmim-Ca1 producing the quinoline $\mathbf{8}$ with a slight improvement (Scheme 4). Even more interesting resulted to prove that this calcium catalyst gave quinoline 9 with almost full conversion (Scheme 4). Nevertheless, this catalyst did not reach the full conversion in the preparation of quinoline 6 (Scheme 4). Consequently, the access to a 'toolbox' of readily available catalysts in order to open the range of substrates is an important characteristic of the work presented here. 


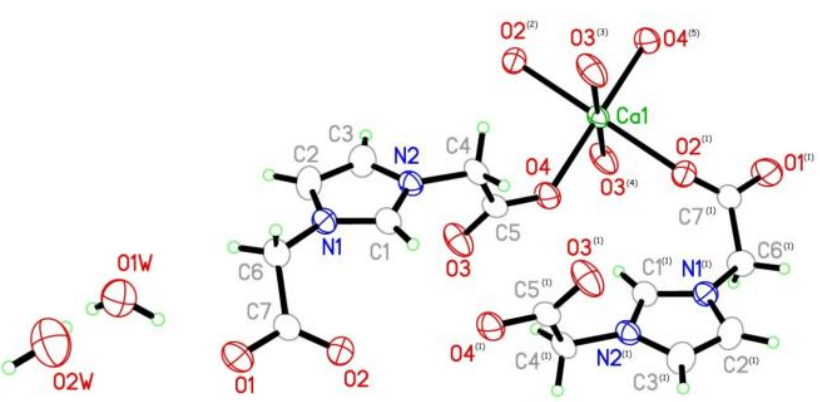

Figure 2. ORTEP plot of bcmim-Ca2; ellipsoids are drawn at $50 \%$ probability level. Symmetry operations: $1-x+1,-y,-z+1 ; 2 x+1, y, z-1 ; 3 x+1, y, z ; 4-x+1$ $-y,-z ; 5-x+2,-y,-z$. Selected bond lengths $(\AA): C a 1-O 2=2.3627(18), C a 1-$ $\mathrm{O}^{(2)}=2.3485(18), \mathrm{Ca} 1-\mathrm{O}^{(3)}=2.3573(19), \mathrm{Ca}^{(-\mathrm{O}} 3^{(4)}=2.3573(19), \mathrm{Ca} 1-\mathrm{O} 4^{(5)}$ $=2.3097(17)$.

This study also reveals the fact that small variations in the precursors of this type of heterogeneous catalysts can modify their activity as observed when comparing the activity of bcmimCa1 with bcmim-Ca2 or bcmim-Ba1 with bcmim-Ba2. In our case, the only difference is the counterion of the organic linker used in the preparation of the material (i.e. chloride or bromide). Thus, we postulate that the halide influences the process of selfassembly that results in the formation of the final structure, packing in different manner. Indeed, their crystallinity differ as observed in the powder XRD analysis (see Supporting Information). Materials bcmim-Ca1 and bcmim-Ba1 have been previously characterized by X-ray diffraction by Dyson and coworkers. ${ }^{[24]}$ Furthermore, material bcmim-Ca2 has been characterized by X-ray diffraction analysis. In compound bcmim-Ca2, there are water molecules per asymmetric unit. Each calcium is bound by six imidazolium anions via one of their carboxylate oxygen atoms (bonding distances in Figure 2). These imidazolium anions act as bridges that link $\mathrm{Ca}$ atoms in layers that extend perpendicular to the $b$ axis (Ca1-Ca1 distance along the a axis: $4.8879(6) \AA$; Ca1-Ca1 distance along the $c$ axis: $7.1117(12) \AA$ ) (Figures 3 and 4). These layers are connected among them by hydrogen bonds with the water molecules present in the crystal. The main difference with bcmim-Ca1 ${ }^{[24]}$ is that halide $(\mathrm{Cl})$ anion is not present in the crystal structure, due that the $\mathrm{Ca}$ coordination sphere is completed with imidazolium anions and crystal charge is compensated these way.

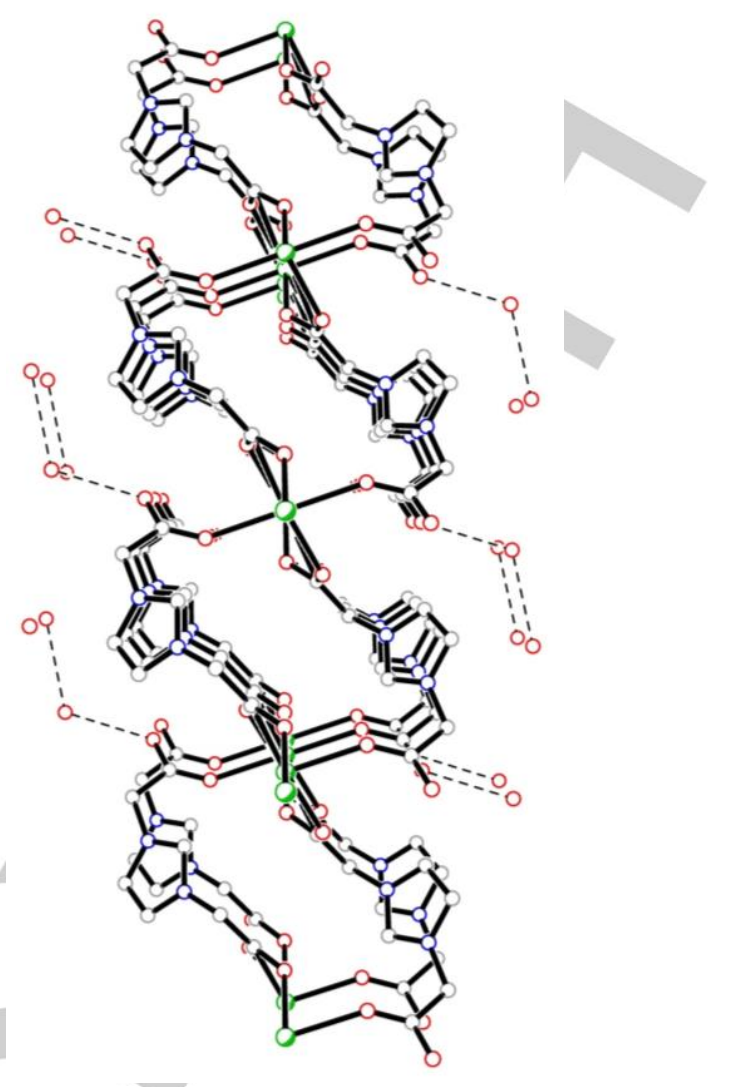

Figure 3. Crystal packing along the a axis for bcmim-Ca2. Hydrogen atoms are omitted for clarity. Color code: $\mathrm{Ca}$ (green), C (grey), O (red), N (blue).



Figure 4. Crystal packing along the $c$ axis for bcmim-Ca2. Hydrogen atoms are omitted for clarity. Color code: Ca (green), C (grey), O (red), N (blue). 


\section{Conclusions}

To conclude, this study has expanded the use of heterogeneous catalysts based on alkaline earth metals (such as calcium and barium). The preparation of a variety of heterogeneous catalysts based on calcium and barium was achieved by means of a straightforward and easy to carry out procedure. This study also showed that small variations in the metal-organic framework precursors, such as the counterion of the organic linker, provided different pattern in the final structure, what can influence in the catalytic activity of the heterogeneous catalyst. The set of prepared catalysts is a toolkit to mediate in synthetic processes, such as the synthesis of quinoline via Friedländer reaction. The catalytic systems presented allow the reaction between carbonyl compounds and not only 2aminobenzophenones but also 2-aminobenzaldehydes, which have not previously been reacted in the presence of metalorganic frameworks for quinoline synthesis. Both, conventional heating or microwave heating can be employed with similar results in this transformation, although longer reaction times are needed for the former. Furthermore, the reusability of this type of catalysts have been proved, the material bcmim-Ba1 being the most robust among the considered in this study.

\section{Experimental Section}

General. All reagents and solvents were used as supplied commercially. Analytical thin-layer chromatography (TLC) was performed on $0.2 \mathrm{~mm}$ coated Science silica gel (EM 60-F254) plates purchased from Merck, Germany. Visualization was achieved by UV light $(254 \mathrm{~nm})$. Flash column chromatography was performed using silica gel and a gradient solvent system (hexane-ethyl acetate as eluent). ${ }^{1} \mathrm{H}$ and ${ }^{13} \mathrm{C}$ NMR spectra were measured on 300 and $400 \mathrm{MHz}$ spectrometers and the residual solvent peak was used as an internal reference: proton (chloroform $\delta 7.26 \mathrm{ppm}$ ) and carbon (chloroform $\delta 77 \mathrm{ppm}$ ). Chemical shifts (ppm) were recorded with tetramethylsilane (TMS) as the internal reference standard. Multiplicities are given as $s$ (singlet), brs (broad singlet), $d$ (doublet), $t$ (triplet), dd (doublet of doublets) or $m$ (multiplet). The number of protons $(\mathrm{n})$ for a given resonance is indicated by $\mathrm{nH}$ and constant coupling are reported as a $J$ value in $\mathrm{Hz}$. The conversion of the reactions and purity of the products were determinate by $\mathrm{GC}$ analysis using a Younglin 6100GC, equipped with a flame ionization detector and a Phenomenex ZB-5MS column (5\% PH-ME siloxane): $30 \mathrm{~m}$ (length), $0.25 \mathrm{~mm}$ (inner diameter) and $0.25 \mu \mathrm{m}$ (film).

General procedure for the synthesis of quinolines. A mixture of the corresponding 2-aminobenzaldehyde (see Supporting Information) or 2aminoarylketone and ketone $\left(2,5\right.$ or 10 equivalents) was stirred at $80{ }^{\circ} \mathrm{C}$ in the presence of the corresponding catalyst (10 mol-\% metal) employing microwaves as the heating source (initial power $80 \mathrm{~W}$ ) or conventional heating. After cooling down the mixture, it was filtered through celite to remove the catalyst, eluting with ethyl acetate. The solvent and the excess of ketone were removed under reduced pressure to provide the crude product, which was purified, if necessary, by flash column chromatography on silica gel using hexane-ethyl acetate as eluent.

Structural analysis of bcmim-Ca2. ${ }^{[27]} \mathrm{X}$-ray diffraction data were measured on a Bruker Smart CCD diffractometer with graphite monochromated Mo Ka radiation $(\lambda=0.71073 \AA)$ at $298(1) \mathrm{K}$. Data collection was based on three $\omega$-scan runs (starting $=-34^{\circ}$ ) at values of $\varphi$ $=0^{\circ}, 120^{\circ}$, and $240^{\circ}$ with the detector at $2 \theta=-32^{\circ}$. For each of the runs, 606 frames were collected at $0.3^{\circ}$ intervals and $10 \mathrm{~s}$ per frame. An additional run at $\varphi=0^{\circ}$ of 100 frames was collected to improve redundancy. The diffraction frames were integrated using the program SAINT, and the integrated intensities were corrected for Lorentz_polarization effects and absorption with SADABS. The structure was solved by direct methods and refined to all 2366 unique $\mathrm{F}_{0}{ }^{2}$ by full matrix least-squares calculations using SHELXL-97. All the hydrogen atoms, except the hydrogens of the $\mathrm{H}_{2} \mathrm{O}$ molecules, were placed at idealized positions and refined using a riding model. The positions of the water hydrogens are located in the Fourier difference map and refined using a riding model. Final R1 indices [1852 with $1>2 \sigma(I)] 0.0527$, wR2 = 0.1163. $R$ indices (all data) $R 1=0.0713$, wR2 $=0.1246$. S[F2] 1.095 for 154 refined parameters. Crystal data: $\mathrm{C} 14 \mathrm{H} 22 \mathrm{CaN} 4 \mathrm{O} 12, \mathrm{M}=478.44$, monoclinic, $\mathrm{a}=4.8879(6) \AA, \mathrm{b}=29.526(4) \AA, c=7.1117(12) \AA, \alpha=y=$ $90^{\circ}, \quad \beta=93.693(2)^{\circ}, \quad V=1024.2(2) \AA^{3}, \quad$ crystal dimensions: $0.32 \times 0.22 \times 0.08 \mathrm{~mm}$, space group: $\mathrm{P} 21 / \mathrm{n}, \mathrm{Z}=2, \mathrm{~F}(000)=500, \mu=0.377$ $\mathrm{mm}^{-1}$.

\section{Acknowledgements}

The Spanish Ministry (Ministerio de Economía y Competitividad, CTQ2015-66624-P) and the University of Alicante (VIGROB285) are gratefully acknowledged for financial support. M.A.S. thanks the Spanish Ministry (Ministerio de Educación, Cultura y Deporte, FPU15/06040) for a Predoctoral Fellowship.

Keywords: alkaline earth $•$ imidazole $\cdot$ heterogeneous catalyst $•$ quinoline $\cdot$ metal-organic framework

[1] P.-Y. Chung, Z.-X. Bian, H.-Y. Pun, D. Chan, A. S.-C. Chan, C.-H. Chui J. C.-O. Tang, K.-H. Lam, Future Med. Chem. 2015, 7, 947-967.

[2] O. Afzal, S. Kumar, M. R. Haider, M. R. Ali, R. Kumar, M. Jaggi, S Bawa, Eur. J. Med. Chem. 2015, 97, 871-910.

[3] (a) J. Marco-Contelles, E. Pérez-Mayoral, A. Samadi, M. C. Carreiras E. Soriano, Chem. Rev. 2009, 109, 2652-2671; (b) A Dhakshinamoorthy, H. Garcia, Chem. Soc. Rev. 2014, 43, 5750-5765 (c) S. M. Prajapati, K. D. Patel, R. H. Vekariya, S. N. Panchal, H. D. Patel, RSC Adv. 2014, 4, 24463-24476.

[4] (a) Z. N. Siddiqui, K. Khan, New J. Chem. 2013, 37, 1595-1602; (b) V. F. Batista, D. C. G. A. Pinto, A. M. S. Silva, ACS Sustainable Chem. Eng. 2016, 4, 4064-4078; (c) S. Ponra, K. C. Majumdar, RSC Adv 2016, 6, 37784-37922.

[5] M. Arrowsmith, M. S. Hill, in Comprehensive Inorganic Chemistry II (Second Edition) (Ed.: K. Poeppelmeier), Elsevier, Amsterdam, 2013, pp. 1189-1216.

[6] (a) J. Y. Lee, O. K. Farha, J. Roberts, K. A. Scheidt, S. B. T. Nguyen, J. T. Hupp, Chem. Soc. Rev. 2009, 38, 1450-1459; (b) A Dhakshinamoorthy, M. Alvaro, H. Garcia, Catal. Sci. Technol. 2011, 1, 856-867; (c) A. Dhakshinamoorthy, M. Alvaro, H. Garcia, Chem. Commun. 2012, 48, 11275-11288; (d) H. Furukawa, K. E. Cordova, M. O'Keeffe, O. M. Yaghi, Science 2013, 341, 974; (e) P. Valvekens, F. Vermoortele, D. De Vos, Catal. Sci. Technol. 2013, 3, 1435-1445; (f) P. Garcia-Garcia, M. Muller, A. Corma, Chem. Sci. 2014, 5, 2979-3007; (g) J. Gascon, A. Corma, F. Kapteijn, F. X. Llabres i. Xamena, ACS Catal. 2014, 4, 361-378; (h) J. Liu, L. Chen, H. Cui, J. Zhang, L. Zhang, C.-Y. Su, Chem. Soc. Rev. 2014, 43, 6011-6061; (i) J. Jiang, O. M. Yaghi, Chem. Rev. 2015, 115, 6966-6997; (j) A. Dhakshinamoorthy, A. M. Asiri, H. Garcia, Catal. Sci. Technol. 2016, 6, 5238-5261; (k) A. Dhakshinamoorthy, A. M. Asiri, H. Garcia, Chem. Eur. J. 2016, 22, 
8012-8024; (I) B. Li, H.-M. Wen, Y. Cui, W. Zhou, G. Qian, B. Chen, Adv. Mater. 2016, 28, 8819-8860; (m) A. M. Ullman, J. W. Brown, M. E. Foster, F. Leonard, K. Leong, V. Stavila, M. D. Allendorf, Inorg. Chem. 2016, 55, 7233-7249; (n) L. Zeng, X. Guo, C. He, C. Duan, ACS Catal. 2016, 6, 7935-7947.

[7] E. Pérez-Mayoral, J. Čejka, ChemCatChem 2011, 3, 157-159.

[8] W.-Y. Gao, K. Leng, L. Cash, M. Chrzanowski, C. A. Stackhouse, Y. Sun, S. Ma, Chem. Commun. 2015, 51, 4827-4829.

[9] N. A. Khan, Z. Hasan, S. H. Jhung, Adv. Porous Mater. 2013, 1, 91-102.

[10] J.-M. Begouin, M. Niggemann, Chem. Eur. J. 2013, 19, 8030-8041.

[11] K. M. Fromm, Coord. Chem. Rev. 2008, 252, 856-885.

[12] D. Saha, T. Maity, R. Sen, S. Koner, Polyhedron 2012, 43, 63-70.

[13] T. Maity, D. Saha, S. Das, S. Koner, Eur. J. Inorg. Chem. 2012, 49144920.

[14] P. Valvekens, D. Jonckheere, T. De Baerdemaeker, A. V. Kubarev, M. Vandichel, K. Hemelsoet, M. Waroquier, V. Van Speybroeck, E. Smolders, D. Depla, M. B. J. Roeffaers, D. De Vos, Chem. Sci. 2014, 5, 4517-4524.

[15] F. Liu, Y. Xu, L. Zhao, L. Zhang, W. Guo, R. Wang, D. Sun, J. Mater. Chem. A 2015, 3, 21545-21552.

[16] A. E. Platero-Prats, M. Iglesias, N. Snejko, Á. Monge, E. GutiérrezPuebla, Crystal Growth \& Design 2011, 11, 1750-1758.
[17] A. E. Platero-Prats, V. A. de la Peña-O'Shea, M. Iglesias, N. Snejko, Á. Monge, E. Gutiérrez-Puebla, ChemCatChem 2010, 2, 147-149.

[18] A. Mallick, E.-M. Schön, T. Panda, K. Sreenivas, D. Díaz-Díaz, R. Banerjee, J. Mater. Chem. 2012, 22, 14951-14963.

[19] A. Allegue, M. Albert-Soriano, I. M. Pastor, Appl. Organomet. Chem. 2015, 29, 624-632.

[20] P. Trillo, I. M. Pastor, Adv. Synth. Catal. 2016, 358, 2929-2939.

[21] M. Albert-Soriano, I. M. Pastor, Eur. J. Org. Chem. 2016, 5180-5188.

[22] O. Kühl, G. Palm, Tetrahedron: Asymmetry 2010, 21, 393-397.

[23] Z. Fei, D. Zhao, T. J. Geldbach, R. Scopelliti, P. J. Dyson, Chem. Eur. J. 2004, 10, 4886-4893.

[24] Z. Fei, T. J. Geldbach, R. Scopelliti, P. J. Dyson, Inorg. Chem. 2006, 45, 6331-6337.

[25] (a) C. L. Diedrich, D. Haase, J. Christoffers, Synthesis 2008, 2199 2210; (b) C. L. Diedrich, D. Haase, W. Saak, J. Christoffers, Eur. J. Org Chem. 2008, 1811-1816.

[26] M. Fujita, Y. J. Kwon, S. Washizu, K. Ogura, J. Am. Chem. Soc. 1994, 116, 1151-1152.

[27] Crystal structure data was deposited at the Cambridge Crystallographic Data Centre, with the following deposition number: CCDC 1550368. 
Entry for the Table of Contents (Please choose one layout)

\section{FULL PAPER}

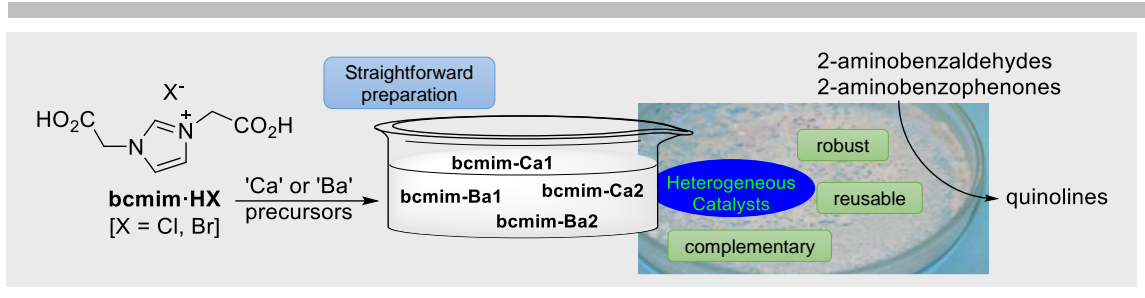

A variety of calcium and barium based heterogeneous catalyst have been straightforward prepared from readily available organic linkers and metal sources. The set of materials constitutes a valuable catalysts array for organic transformations, opening up their practicability.

\section{Alkaline earth catalyst ${ }^{\star}$}

María Albert-Soriano, Paz Trillo, Tatiana Soler, Isidro M. Pastor*

Page No. - Page No.

Versatile barium and calcium imidazolium-dicarboxylate heterogeneous catalysts in quinoline synthesis

*one or two words that highlight the emphasis of the paper or the field of the study 